\title{
Assessment of hemostatic function in pregnant women undergoing surgery using a Platelet Function Analyzer-100
}

\author{
Nan-Seol Kim and Dong-Hun Lee \\ Department of Anesthesiology and Pain Medicine, Soonchunhyang University Hospital, College of Medicine, Soonchunhyang \\ University, Cheonan, Korea
}

In general, normal pregnancy is considered as a hypercoagulable state [1], and this is probably a protective adaptation to lessen the risks associated with acute hemorrhage at delivery. However, pre-eclampsia is associated with a risk of abnormal hemostasis, which occurs most commonly secondary to thrombocytopenia [2]. Of the numerous studies, regarding hemostatic functions in pregnancy, routine coagulation tests, such as platelet ctount, prothrombin time (PT), activated partial thromboplastin time (aPTT), and bleeding time, do not accurately reflect the blood coagulation status of pregnant women [2]. However, Platelet Function Analyzer-100 (PFA100, Dade-Behring, Marburg, Germany), an in vitro analyzer of platelet function, may be of value. The purpose of this study was to evaluate the physiologic changes in the coagulation systems, in healthy pregnant and pre-eclamptic women in Korea using PFA-100.

This study was approved by the Institutional review board (IRB) at our institution, and written informed consent was obtained from each patient. We enrolled 175 American Society of Anesthesiologists physical status classification 1 or 2 women, who were scheduled for surgery and were divided into three groups, healthy non-pregnant women (Group A), healthy pregnant women (Group B) and pre-eclamptic women (Group C). Blood samples were obtained through a 16-gauge needle at venous cannulation on admission for surgery. Routine hematologic and coagulation tests were performed, including platelet count, hematocrit (Hct), activated partial thromboplastin times (aPTT), and prothombin time (PT). For
PFA-100 analysis, $2 \mathrm{ml}$ of citrated whole blood was pipetted into a test cartridge, containing epinephrine as a platelet activator, and CT measured according to the respective guidelines. All results are expressed as the mean $\pm \mathrm{SD}$, and where appropriate, the differences between the sample means are presented with $95 \%$ confidence interval (CI). Comparisons were also made, using an analysis of variance (ANOVA). If a significant difference was noted, Bonferroni multiple comparison test was used to determine the intergroup differences. Student's t-test or chi-square tests were used to compare the variables between the groups, where appropriate.

No significant differences in age, height, and blood groups were found among the three groups. However, the average weight of women in Group B and Group C was significantly higher than that of Group A. The mean platelet count was significantly reduced in Group B and Group C, compared with Group A. Other clotting tests, including PT and aPTT, were of no statistical difference among the three groups, and remained as the reference ranges. The mean PFA-100 CT, the time taken to form an effective platelet plug, of group B was shorter than that of Group A (98.1 vs 118.8, P < 0.05). However, the mean PFA$100 \mathrm{CT}$ was significantly prolonged in Group C compared with Group B (132.2 vs 98.1, P < 0.05). Additionally, the mean PFA100 CT was significantly prolonged in Group C compared with Group A (Table 1).

PFA-100 has been validated against clinical and laboratory tests and found to be as sensitive and specific, as platelet aggregometry, the current gold standard [3]. Also, it is the whole

Corresponding author: Nan-Seol Kim, M.D., Department of Anesthesiology and Pain Medicine, Soonchunhyang University Hospital, College of Medicine, Soonchunhyang University, 23-20, Bongmyeong-dong, Cheonan 330-100, Korea. Tel: 82-41-570-2722, Fax: 82-41-573-3559, E-mail: nskim1977@naver.com

(c) This is an open-access article distributed under the terms of the Creative Commons Attribution Non-Commercial License (http:// creativecommons.org/licenses/by-nc/3.0/), which permits unrestricted non-commercial use, distribution, and reproduction in any medium, provided the original work is properly cited. 
Table 1. Comparison of the Results of PFA-100 CT

\begin{tabular}{|c|c|c|c|c|c|}
\hline \multirow{2}{*}{$\mathrm{CT}(\mathrm{sec})$} & \multirow{2}{*}{ Mean \pm SD } & \multicolumn{2}{|c|}{ 95\% Confidence interval for mean } & \multirow{2}{*}{ Minimum } & \multirow{2}{*}{ Maximum } \\
\hline & & Lower & Upper & & \\
\hline Group A $(n=55)$ & $118.8 \pm 30.6^{*}$ & 110.5 & 127.1 & 86 & 300 \\
\hline Group B $(\mathrm{n}=55)$ & $98.1 \pm 24.8$ & 91.4 & 104.9 & 54 & 213 \\
\hline Group C $(\mathrm{n}=55)$ & $132.2 \pm 44.5^{*, \dagger}$ & 119.9 & 144.0 & 72 & 300 \\
\hline
\end{tabular}

Values are expressed as mean \pm SD. PFA-100 CT: platelet function analyzer-100 closure time. Group A: control group, Group B: healthy pregnant women, Group C: pre-eclamptic women. ${ }^{*} \mathrm{P}<0.05$ compared with Group B. ${ }^{\dagger} \mathrm{P}<0.05$ compared with Group A and Group B.

blood test, which is able to measure the ability of platelets to occlude a vascular breach, whereas the platelet aggregation test evaluates only the platelets in the plasma [4], and this test is easy to perform, gives reproducible results, and can test relatively small volumes $(0.8 \mathrm{ml})$ of the citrated blood [5]. However, it is well known that PFA-100 CT has some limitations. Despite having $98 \%$ negative and $94 \%$ positive predictive values, this test is not always sensitive to all platelet function defects. In addition, PFA-100 is sensitive to many variables, such as Hct, platelet count, drug and dietary effects, sampling time and $\mathrm{ABO}$ blood group [5].

Using PFA-100, it was found that in patients with pregnancyinduced thrombocytopenia, platelet function might be preserved when the platelet count is as low as $60,000 / \mathrm{mm}^{3}$, unless anemia was present [4]. Another study showed that in the platelet count below $80,000 / \mathrm{mm}^{3}$, platelet number begins to influence CT, causing it to lengthen [5]. Therefore, in the present study, women with anemia (Hct $<30 \%)$ and thrombocytopenia (platelet count $<80,000 / \mathrm{mm}^{3}$ ) were excluded.

The normal limits of CT are hospital specific. Although our hospital has reference ranges for the general population, the range of the population of pregnant women has not yet been determined. The mean CT of healthy pregnant women is lower than that of the nonpregnant population (98.1 vs $118.8 \mathrm{sec}, \mathrm{P}<$ 0.05 ), suggesting increased primary hemostatic capacity (Table 1). On the other hand, PFA-100 CT of pre-eclamptic women was significantly prolonged than that of the healthy pregnant group (132.2 vs 98.1, $\mathrm{P}<0.05)$. In addition, the mean CT of pre-eclamptic women exceeded the $95 \%$ reference interval of the healthy control group. This result provides evidence of impairment of primary hemostatic function in pre-eclamptic women.

The limitations of this study should be addressed. First, we did not perform the whole blood platelet aggregometry test, which is the gold standard for detecting platelet function defects. Furthermore, comparison between the mild and severe forms of pre-eclampsia was not conducted.

In conclusion, the results of this study, consistent with the previous studies, revealed that healthy term pregnant women in the hypercoagulable state showed decreased PFA-100 CT, but pre-eclamptic women showed significantly prolonged PFA-100 CT.

\section{References}

1. Cerneca F, Ricci G, Simeone R, Malisano M, Alberico S, Guaschino S. Coagulation and fibrinolysis changes in normal pregnancy. Increased levels of procoagulants and reduced levels of inhibitors during pregnancy induce a hypercoagulable state, combined with a reactive fibrinolysis. Eur J Obstet Gynecol Reprod Biol 1997; 73: 316.

2. Sharma SK, Philip J, Whitten CW, Padakandla UB, Landers DF. Assessment of changes in coagulation in parturients with preeclampsia using thromboelastography. Anesthesiology 1999; 90: 38590.

3. Mammen EF, Comp PC, Gosselin R, Greenberg C, Hoots WK, Kessler CM, et al. PFA-100 system: a new method for assessment of platelet dysfunction. Semin Thromb Hemost 1998; 24: 195-202.

4. Vincelot A, Nathan N, Collet D, Mehaddi Y, Grandchamp P, Julia A. Platelet function during pregnancy: an evaluation using the PFA100 analyser. Br J Anaesth 2001; 87: 890-3.

5. Harrison P. Platelet function analysis. Blood Rev 2005; 19: 111-23. 